Sociologie et sociétés

\title{
Surveiller les flux, contrôler les hommes
}

Du travail et de sa division dans l'industrie chimique et nucléaire

\section{Monitoring flows, controlling humans} Of work and its division in the chemical and nuclear industry

\section{Gwenaële Rot et François Vatin}

Volume 48, numéro 1, printemps 2016

Le travail au prisme de l'activité

Work through the lens of activity

URI : https://id.erudit.org/iderudit/1036885ar

DOI : https://doi.org/10.7202/1036885ar

Aller au sommaire du numéro

Éditeur(s)

Les Presses de l’Université de Montréal

ISSN

0038-030X (imprimé)

1492-1375 (numérique)

Découvrir la revue

Citer cet article

Rot, G. \& Vatin, F. (2016). Surveiller les flux, contrôler les hommes : du travail et de sa division dans l'industrie chimique et nucléaire. Sociologie et sociétés, 48(1), 97-116. https://doi.org/10.7202/1036885ar
Résumé de l'article

Comprendre en quoi et comment une activité, dite de travail, est productive et socialement reconnue comme telle, est indispensable pour penser les modes d'encadrement social de cette activité, au niveau de l'atelier, de l'entreprise, de la société tout entière. Encore faut-il pour cela saisir le travail dans sa technicité. Telle est la démarche que nous allons développer ici, en partant d'une analyse du travail de surveillance-contrôle observé dans différents sites chimiques et nucléaires pour penser, les problèmes de l'organisation salariale dans ce type d'entreprises.
Tous droits réservés @ Les Presses de l’Université de Montréal, 2016

Ce document est protégé par la loi sur le droit d'auteur. L’utilisation des services d'Érudit (y compris la reproduction) est assujettie à sa politique d'utilisation que vous pouvez consulter en ligne.

https://apropos.erudit.org/fr/usagers/politique-dutilisation/ 


\section{Surveiller les flux, contrôler les hommes}

Du travail et de sa division dans l'industrie chimique et nucléaire

\section{GWENAËLE ROT}

Centre de sociologie des organisations (CSO-CNRS) Sciences-Po Paris

Courriel: gwenaele.rot@sciencespo.fr

\section{FRANÇOIS VATIN}

Institutions et dynamiques historiques de l'économie et de la société (IDHES-CNRS) Université de Paris Ouest

Courriel:vatin@u-parisı.fr

Quand vous montez une voiture, vous prenez une portière, vous la mettez en place, vous mettez une goupille et cela repart [il claque des mains] et, à côté, l'autre, il monte la serrure,

terminé [il claque des mains]. Cela se voit, cela se touche. Nous, qu'est-ce qu'on a?

On ne voit que des tuyaux et il passe un produit dedans qu'on ne voit jamais. Il faut arriver à s'imaginer ce qui peut se passer... Les stocks, on ne voit rien, on voit les niveaux qui montent, qui descendent. On est obligé de se visualiser la production dans la tête. On s'imagine ce qui peut se passer. Le jour où on commence à voir le produit, cela veut dire que cela sort dehors. Or, il faut savoir que c'est cancérigène, que c'est explosif. (agent de maîtrise posté, ancien opérateur, chimie)

$\mathrm{D}$

ÉCRIRE LE TRAVAIL DANS UNE INDUSTRIE DE FLUX, usine chimique ou centrale nucléaire, est un exercice délicat. La transformation chimique de la matière reste passablement obscure pour le non-spécialiste; quant à la production d'électricité, elle a un caractère mystérieux, un peu magique. Ces processus industriels sont d'autant moins accessibles au sens commun qu'ils ne sont pas directement «visibles». Un autre élément de brouillage cognitif s'ajoute à cela: il est difficile de rattacher cet univers aux représentations communes du travail. En effet, il n'y a pas d'équivalent aux Temps modernes de Charlie Chaplin pour fournir une représentation, même caricaturale, du 
travail dans ce type de monde productif, aux antipodes du modèle canonique de la répétition machinique du geste ${ }^{1}$. Même le fabuleux film d'Alain Resnais sur la production de matière plastique de 1958, Le Chant du styrène, pourtant agrémenté d'un poème didactique, bien qu'en alexandrins, de Raymond Queneau ${ }^{2}$, ne suffit pas à rendre familier un tel univers.

Dans cet article, nous souhaitons montrer en quoi la «boîte noire» du travail, verrouillée par un ciment idéologique solide, vieux de deux siècles, mérite d'être rouverte, à la lumière du cas des industries de flux. Dans la poursuite de Pierre Naville, nous avions abordé cette question dans les années 1980 (Galle et Vatin, 1980, 1981; Vatin, 1987). Nous y revenons aujourd'hui à la lumière de nouvelles enquêtes (Rot et Vatin, à paraître). Dans ce contexte, s'intéresser, comme nous allons le faire, à l'activité de travail, ici, dans l'industrie de flux, ne consiste aucunement à ouvrir un chapitre de plus dans le catalogue des intérêts des sociologues du travail. Le «travail-activité» n'est pas un concept nouveau, car le travail, en soi, est activité et rien d'autre (Bidet et Vatin, 2016) ${ }^{3}$. Mais il s'agit (c'est sa propriété caractéristique au sens mathématique de l'expression) d'une activité à vocation productive. Comprendre en quoi et comment une activité, dite de travail, est productive et socialement reconnue comme telle, est indispensable pour penser les modes d'encadrement social de cette activité, pour ce qui est de l'atelier, de l'entreprise, de la société tout entière (Vatin, 2014). Encore faut-il pour cela comprendre le travail dans sa technicité. Telle est la démarche que nous allons développer ici, en partant d'une analyse du travail de surveillance-contrôle observé dans différents sites chimiques et nucléaires pour penser, dans un second temps, les problèmes de l'organisation salariale dans ce type d'entreprises ${ }^{4}$.

1. Dont l'automobile reste encore le secteur emblématique comme le suggère la citation mise en exergue. Sur le travail dans l'automobile et ses représentations voir Rot, 2006.

2. Sur la représentation de l'automation dans ce film, voir Rot, 2007.

3. Le regretté François Sigaut, spécialiste d'ethnologie des techniques, s'était inquiété un jour de l'émergence de cette nouvelle catégorie: «Pourquoi préciser le travail-activité? Le travail peut-il être autre chose?» La remarque est fine; c'est par abus de langage que l'on parle, par exemple, de «marché du travail». Comme Marx l'avait souligné, on ne saurait vendre ou acheter le travail. L'entrepreneur n'achète qu'une «capacité» ou «force» de travail. Il loue, pendant un temps limité, la disponibilité des forces manuelles et intellectuelles des salariés.

4. Cette démarche s'inscrit explicitement en opposition à la thèse, qui, au début des années 1980, dénonçait le «déterminisme technologique» dans la sociologie du travail (Maurice, 1980). La démarche développée au sein du Laboratoire d'économie et de sociologie du travail d'Aix-en-Provence (LEST) visant à mettre en évidence un «effet sociétal» (national), indépendamment des variables économiques et technologiques (Maurice, Sellier et Silvestre, 1982), a un caractère tautologique. Elle repose en effet sur la comparaison d'établissements similaires dans deux pays différents, laquelle met en évidence des différences résiduelles dans l'organisation du travail, sans pour autant prouver que les conditions techno-économiques de la production ne seraient pas au moins aussi déterminantes que cet «effet sociétal». Cette démarche a également été mise en œuvre par Duncan Gallie (1977 et 1978) dans une comparaison de deux raffineries de pétrole en France et en Grande-Bretagne. 


\section{Enquêtes}

Les enquêtes sur lesquelles s'appuie cet article ont été réalisées en 2007 dans deux usines chimiques et en 2009 dans une centrale nucléaire. D’une durée de deux semaines complètes par site, ces enquêtes, ont combiné entretiens, observation in situ et analyses documentaires (rapport d'incident, comptes rendus de réunion, cahiers de quart). Une quinzaine d'entretiens semi-directifs ont été réalisés dans chacun des sites auprès de salariés: opérateurs intérieurs et extérieurs, chefs de poste, membres de l'encadrement de jour, ingénieurs et chefs de service. Ces entretiens ont porté sur les trajectoires professionnelles, les conditions concrètes de l'apprentissage, le déroulement des activités en situation «normale » et en situation plus critique, les processus de décision individuelle et collective dans le cours de l'action, les relations de travail entre pairs ainsi qu'avec la hiérarchie intermédiaire et supérieure. Une partie des questionnements s'adossait aux observations de l'activité réalisées sur le terrain: suivis de rondes sur les équipements (le long des canalisations, en salle des machines), de la conduite en salle de contrôle des installations, des moments de transmission de consignes lors de la relève entre équipes, des réunions d'équipes postées, des réunions de planification de travaux. Pendant les quarts de nuit, nous avons également participé aux repas organisés par l'équipe qui nous accueillait.

Soulignons que ces enquêtes ont porté sur le personnel propre des entreprises et non sur le personnel sous-traitant de maintenance et alors que ces sites industriels étaient en fonctionnement ordinaire et non en arrêt périodique. Par ailleurs, le présent article ne vise pas à analyser en détail l'organisation du travail propre à chacun des établissements enquêtés, ni les formes des relations professionnelles (syndicalisme, conflits sociaux) qui s'y nouent. Il s'agit, à partir du matériau recueilli, de s'interroger sur la signification sociale de ce mode particulier de travail que constitue la fonction de surveillance-contrôle. C'est pourquoi aussi, nous n'avons pas cherché à mettre en évidence les différences entre les situations respectives des industries chimique et nucléaire, même si nous les notons à l'occasion. Ce qui nous intéresse ici, c'est bien la similarité très marquée entre ces secteurs, si on les examine du point de vue du contenu même de l'activité productive.

\section{LA FONCTION DE SURVEILLANCE-CONTRÔLE, EMBLÉMATIQUE DU TRAVAIL CONTEMPORAIN}

La notion de «travail» fait, de façon conjointe, l'objet de deux représentations, épistémologiquement contradictoires, mais qui pourtant convergent dans le discours sociopolitique et même sociologique le plus courant (Vatin, 2008). La première, la plus ancienne, conçoit le travail sur une base énergétiste: il y a «travail», parce qu'il y a dépense de force. Ainsi considérée, la notion de travail s'applique à l'usage productif de l'homme, mais aussi des animaux, voire des forces naturelles et des machines. C'est 
parce qu'il est ici défini comme dépense de force, que le travail fatigue, au sens physique du terme (on parle aussi de la fatigue des mécanismes) et qu'il est donc peine, au sens moral. La seconde représentation du travail prend appui sur la première, via la tradition de l'économie politique occidentale, héritière de la morale judéo-chrétienne de la chute et de la rédemption. Puisqu'il faut payer pour obtenir des personnes du travail, c'est que celui-ci est une utilité négative, une désutilité selon la formule de l'économiste néoclassique britannique William Stanley Jevons. Par le biais de l'équilibre marchand, on retrouve la figure de la peine, théorisée par Marx par le concept d'aliénation ${ }^{5}$. Pensé sous le registre du marché, le travail est ainsi ramené au salariat.

Ces deux définitions sont, dans leur construction, on ne peut plus dissemblables. La première aborde le travail sur une base naturaliste. Comme dépense de force, le travail est une catégorie transhistorique. La seconde, au contraire, fait du travail une catégorie historique qui n'apparaîtrait qu'avec la généralisation de la société marchande au XVIII ${ }^{\mathrm{e}}$ siècle (Méda, 1995). Pourtant, ces deux représentations convergent pour faire du travail une «peine». Dans le premier cas, la peine a un fondement physiologique, dans le second, elle est le produit de l'aliénation constitutive de la relation salariale. Cette seconde représentation peut alors absorber la première, laquelle n'est plus guère explicitement formulée, mais reste pourtant sous-jacente aux représentations dominantes du travail, profanes comme savantes. Défini comme une désutilité par la théorie économique, le travail est dépense, fatigue, peine, usure. Au cours du $\mathrm{XIX}^{\mathrm{e}}$ siècle, les sciences du travail s'efforceront de mesurer cette fatigue en termes énergétiques (Vatin, 1993, 1999). Au xx ${ }^{\mathrm{e}}$ siècle, alors que, avec la mécanisation, finit par s'imposer le constat que l'usage productif de l'homme est de moins en moins «physique», on chercha, aussi, à évaluer le travail «intellectuel» comme une dépense de force, ce dont témoigne, par exemple, la catégorie de «charge mentale» des ergonomes. Le raisonnement, qui ramène le travail à la fatigue ou à un quelconque de ses synonymes est en fait circulaire: selon un schéma qui prend sa source dans l'économie politique classique, le travail est par essence «fatigant», puisqu'il n'y aurait pas de sens à le payer si tel n'était pas le cas. Un tel schéma conceptuel évince de l'investigation toute réelle analyse de «l'activité de travail», puisque la fatigue que le travail est postulé générer constitue une propriété suffisante pour caractériser cette activité. C’est oublier, par exemple, qu'à mesure que le travail a moins mobilisé la corporéité des personnes, on a vu se développer des activités sportives, visant, précisément, à «fatiguer» (au sens physiologique du terme) un organisme devenu insuffisamment sollicité par le travail. D’un autre côté, en définissant le travail par le salariat, on a réduit le travail au temps et à l'espace couverts par le contrat de travail, en faisant l'hypothèse que, parce que les personnes sont, juridiquement, «au travail» (dans le temps et l'espace couverts par le contrat de travail), elles travaillent effectivement et, inversement, qu'elles ne travaillent

5. Pour Marx, le travail est, dans la société salariale, aliéné au sens où le travailleur en est dépossédé par la vente de sa «force de travail». Mais pour Marx, à l'inverse du schéma que nous discutons ici, l'aliénation ne définit pas le travail, qui est, dans sa substance ontologique, «libre jeu des forces naturelles de l'homme». 
jamais dans un autre contexte spatio-temporel, hypothèse qui ne résiste pas à l'observation ordinaire de nombre de configurations professionnelles.

A contrario de ce double système de représentation, nous posons ici que ce qui définit le travail est son caractère productif. Une activité n'est «travail» que parce qu'elle est reconnue productive et cela, quelles que soient les instances et les modalités, plus ou moins combinées, par lesquelles cette reconnaissance a lieu: par le travailleur luimême, par ses pairs, par l'employeur qui paye le salaire du travail, par les consommateurs qui achètent les produits du travail, etc. L'étude de «l'activité de travail» a alors précisément pour fonction d'identifier en quoi ce que font les gens est productif.

Or, depuis la fin du XIx ${ }^{\mathrm{e}}$ siècle, l'émergence de la fonction de surveillance-contrôle a mis cette question en évidence sur un nouveau mode. Habitués à la représentation du travail-peine, en un temps où les forces physiques de l'homme étaient encore massivement mobilisées, les observateurs ont immédiatement été frappés par cette nouvelle figure paradoxale du travailleur dont la fonction est de ne «rien faire» tant que la production suit son cours, ou, plus précisément, de rester vigilant, c'est-à-dire de s'assurer que la production suit bien son cours. Ainsi l'historien et économiste Georges d'Avenel présentait en 1895 en ces termes le travail de l'ouvrier d'imprimerie: «L'opération s'accomplit toute seule. Un unique ouvrier y assiste, accoudé contre un bâti; il se penche parfois sur un cylindre, examine le papier, serre un écrou, verse un peu d'huile, puis rentre dans son immobilité, type expressif du travail moderne» (Avenel, 1895, p. 548). Georges d'Avenel écrit alors que s'accomplit la «seconde révolution industrielle», caractérisée par la genèse de l'énergie électrique, celle du moteur à explosion et le développement du raffinage pétrolier qui s'ensuit, ainsi que par l'émergence de la grande industrie chimique. Dans ces industries, caractérisées par ce que Pierre Naville a appelé en 1963 le «principe de production chimique», la production résulte des réactions des réactions des matières entre elles (Naville, 1963; Vatin, 1987). L'homme intervient en amont et en aval du circuit de tubulures. Il doit entretenir le système machinique lors d'arrêts où toute production cesse. En temps ordinaire, il doit s'assurer que les choses suivent leur cours. Cette configuration productive a retenu l'attention des premiers sociologues du travail, dès la fin des années 1940, de part et d'autre de l'Atlantique.

La thématique de l' «automation», néologisme forgé par un ingénieur des usines Ford pour designer la manutention automatique («automatic motion»), se diffuse, dès le début des années 1950, dans le monde des grandes entreprises industrielles, via, notamment, la publication d'un petit ouvrage de John Diebold (Diebold, 1952). En 1954, le spécialiste américain du management Peter Drucker distinguait trois modes industriels de production: le "produit unique» (comme le chantier du bâtiment traditionnel), la «série» (emblématisée par l'usine automobile) et le "processus», correspondant à ce que nous avons désigné supra par «industries de flux» (Drucker, 1954). Il conclut que ces dernières industries sont prêtes pour l'automation. À la même époque, le sociologue français Alain Touraine ne disait pas autre chose (Touraine, 1955; Rot, Vatin, 2015). S’il construit sa célèbre typologie en trois «phases» des formes 
du travail ouvrier à partir du cas de l'industrie automobile et plus précisément des usines Renault, c'est avec les exemples du raffinage pétrolier ou de la production hydroélectrique qu'il illustre la «phase C», celle de la «recomposition du travail ${ }^{6} »$. Sa typologie, construite différemment de celle de Drucker, se rapproche d'ailleurs de celle-ci .

C'est à cette époque que commencent à paraître les premiers travaux sociographiques sur le travail dans ce type de configurations productives. Longtemps, en effet, l'industrie chimique a fait figure d'industrie de main-d'œuvre, occupant un large personnel de manœuvres chargés de la manutention de produits dangereux. Le développement de l'usine-tuyau a fait tout à la fois disparaître ce travail de manutention et émerger la nouvelle figure emblématique du contrôleur de flux. En 1956, William Foote Whyte fournit une des premières enquêtes de sociologie industrielle sur le travail de surveillance-contrôle dans une raffinerie de pétrole, travail inspiré par ses observations datant de 1941 (Whyte, 1956 et 1994). Cette thématique devient centrale aux débuts des années 1960 en France comme aux États-Unis. En France, la Commission du conseil supérieur de la recherche scientifique et du progrès technique commande au sociologue Georges Friedmann une grande enquête sur l'automation, dont Pierre Naville prend la direction. Il publie en 1961 son rapport d'enquête et en 1963 un ouvrage de synthèse: Vers l'automatisme social? (Naville, 1961 et 1963). L' "automatisme social», tel que le conçoit Naville, correspond à la dynamique de «chimisation de l'industrie», qui se manifeste avec le développement des industries de flux. Si l'enquête sur laquelle il s'appuie a porté sur un grand nombre de secteurs industriels, le raffinage pétrolier constitue indiscutablement son exemple emblématique. Aux ÉtatsUnis, Robert Blauner publie en 1964 une vaste synthèse à certains égards comparable à celle de Naville sous le titre Alienation and Freedom. The factory workers and his industry (Blauner, 1964). Blauner n'a pas pris connaissance des travaux de Naville, mais il cite en revanche l'ouvrage de Touraine ${ }^{8}$ ainsi que l'article de Whyte.

Le titre de l'ouvrage de Blauner est étonnamment similaire de celui de la troisième partie de celui de Naville: «Automation et aliénation». Cette convergence permet de mettre en évidence la thématique centrale du débat de l'époque. La problématique de l'aliénation renvoie clairement chez ces deux auteurs à une discussion de la théorie de Marx relativement à la réalité du travail automatisé. Le découplage entre le temps des hommes et celui des machines, caractéristique de l'automation, que met en évidence Naville, fournit-il la promesse d'une libération du travail? Conduit-il au contraire à

6. Cette idée de «recomposition» signe la source hégélienne du modèle de Touraine, analogue en ce sens à celui de Lewis Mumford, qu'il cite pour s'en dissocier, lequel distinguait de même, en 1934, les phases éotechnique, paléotechnique et néotechnique (Mumford, 1934/1950).

7. Il en est de même de celle de Joan Woodward (1965), qui cite Drucker. Les typologies de Drucker et Woodward different toutefois de celle de Touraine (et a fortiori de celle de Mumford), en ce que, plus synchroniques que diachroniques, elles n'ont pas son caractère téléologique hégélien. Il faut enfin citer la typologie de James R. Bright (1958), qui inspira Pierre Naville, laquelle organise toute l'histoire des formes industrielles sur un unique axe qui mène à l'automation.

8. L'ouvrage de Touraine est également cité de manière élogieuse dans l'étude de Richard Walker (1957), qui porte sur l'automatisation dans une usine sidérurgique. 
des formes nouvelles d'aliénation? Ce qui est certain en tout cas, c'est que l'observation du travail automatisé impose un renouvellement de la pensée. Il conduit à faire exploser l'apparente cohésion entre les deux représentations communes du travail exposées précédemment: le travail, dépense de force, et le travail, sujétion salariale. Pour aborder les questions classiques des sociologues du travail (la hiérarchie salariale, les modes de management, les conflits socio-organisationnels), il faut revenir au contenu de l'activité elle-même. Que fait un surveillant-contrôleur? En quoi et à quelles conditions son activité est-elle «productive» ? Comment se distribue le travail, pas seulement au sens sociopolitique de la hiérarchie des tâches, mais bien aussi en fonction des contraintes propres de telles organisations productives? Nous allons aborder cette question en nous penchant successivement sur les deux axes qui structurent la division, tout à la fois technique et sociale, du travail dans ces entreprises ${ }^{9}$ : celui qui oppose les travailleurs «du dedans» (les «tableautistes») à ceux «du dehors» (les « opérateurs extérieurs»); celui qui oppose les travailleurs « en quart» à ceux «à la journée».

\section{LE DEDANS ET LE DEHORS: DUALITÉ ET COOPÉRATION DANS LA SURVEILLANCE}

Dans les industries de flux, la surveillance s'effectue à la fois du «dedans», par les opérateurs «intérieurs», installés dans les salles de contrôle, et du "dehors", sur les installations elles-mêmes par les opérateurs «extérieurs». Dans tout site industriel fonctionnant en flux continu, la salle de contrôle constitue le centre névralgique de l'usine. Dans cet espace confiné, protégé des installations plus ou moins bruyantes et malodorantes, qui, elles, sont exposées aux intempéries, des opérateurs intérieurs, également appelés tableautistes, pupitreurs ou consolistes, surveillent la production à partir de consoles informatiques, qui, chacune, couvre un segment des installations. Ils "prennent les commandes», comme on le dit pour une automobile ou un avion $^{10}$.

L'activité des tableautistes est similaire dans les trois sites que nous avons étudiés. Chaque opérateur y surveille sur son écran sa zone de responsabilité, pour laquelle il est seul habilité à modifier les paramètres de fonctionnement. Pour parcourir son territoire, il doit «cliquer» sur les vues des installations qui figurent sur l'écran (fours, colonnes de distillation, bacs de stockage, réacteurs), chacune identifiée par un numéro. Il en fait ainsi apparaître l'état détaillé de fonctionnement. En marche normale, il doit vérifier que les valeurs numériques restent dans les bornes de la norme

9. Rappelons que notre enquête ne porte que sur le personnel propre de ces entreprises et non sur le personnel sous-traitant assurant notamment les tâches de maintenance. Sur le travail d'entretien soustraité dans le nucléaire, voir notamment Doniol-Shaw et al., 1995; Fournier, 2012; sur l'organisation du travail à l'occasion d'arrêts périodiques, dans une perspective d'une comparaison franco-américaine(Bourrier, 1999).

10. L'image de la conduite d'avion ou d'automobile a été à plusieurs reprises utilisée par nos interlocuteurs dans les usines. L'ergonomie des salles de contrôle a d'ailleurs été souvent comparée à celle des cockpits d'avion. 
prescrite, ou, si une opération est en cours, suivre le passage à la nouvelle valeur désirée. En fonction de ses observations, il procède, si nécessaire, à des corrections de paramètres à partir de son clavier ou à des interventions à distance (ouverture ou fermeture de vannes, déclenchement de pompes).

Cette activité engage peu la corporéité, tant en raison de la distance par rapport au produit fabriqué que du caractère physiquement minimal des gestes matériels de travail, qui se résument, pour l'essentiel, au maniement d'un clavier. On échappe ici à la définition idéal-typique de l'ouvrier comme «l'homme qui travaille de ses bras à la production industrielle», posée par Maurice Halbwachs en 1913 (Halbwachs, 1913, p. 59). Produit de l'inertie historique et des traditions syndicales, la culture ouvrière demeure pourtant vivante dans ces industries. La confrontation entre la nature concrète de l'activité et l'imaginaire ouvrier n'est pas d'ailleurs sans provoquer un sentiment de culpabilité qui s'exprime dans un discours contourné sur le travail. L'assimilation canonique du travail à la peine constitue pour ces opérateurs un obstacle épistémique: si nous produisons, c'est que nous travaillons, si nous travaillons, c'est que nous peinons. Mais les «autres» (dans la situation qui suit, les ouvriers d'entretien salariés par les entreprises sous-traitantes) auraient du mal à concevoir la nature particulière de leur peine:

Les gens des entreprises extérieures, pour eux, les gens de l'usine [le personnel salarié par l'entreprise donneuse d'ordres], c'est tous des glandeurs, ils ne foutent rien. Certains n'arrivent pas à comprendre qu'avec un ordinateur, le boulot qu'on a, on n'est pas toujours en train de courir, on n'est pas en train d'ouvrir des vannes, on n'est pas des maçons, on ne monte pas de murs, nous, mais, par contre, quand on met le doigt sur une touche, la conséquence peut être énorme par rapport au travail qu'ils font. Passer huit heures à regarder un ordinateur sans qu'il ne se passe rien, à acquitter des alarmes et tout... Les gens ne se rendent pas compte de la fatigue que cela engendre, ce n'est pas la même fatigue qu'une fatigue musculaire... Des fois, ils disent qu'on fout rien. Et eux, ils montent les escaliers, les échafaudages et c'est leur boulot qui est comme cela. Moi, mon boulot, c'est je suis payé pour regarder un ordinateur, donc, je le regarde. C'est vrai que cela ne fait pas très dynamique; n'empêche que, c'est mon boulot de faire cela. (tableautiste, chimie)

Trois registres argumentaires sont ici combinés pour définir le travail et son utilité: la puissance de l'action (un simple doigt sur une touche peut avoir des conséquences considérables), la fatigue nerveuse qui se substituerait à la fatigue physique, et, enfin, la contrainte salariale (cette activité est un travail puisque c'est pour l'accomplir que je suis payé). Le geste physique minimal accompli (la manipulation des touches du clavier) est alors valorisé comme une prouesse technique: «Entre un organe et un autre, cela ne réagit pas pareil. Je donne un exemple: on a un bouton + et - sur un organe; on appuie comme cela, cela va baisser de 8 , et, sur un autre, le même bouton, on touche à peine, et cela va vite...» (tableautiste nucléaire). La beauté du geste se lit aussi dans le résultat, celui, abstrait, des courbes retraçant l'état des variables de fabrication:

Lundi, j'ai remonté à $100 \%$. J'ai sorti deux fois l'alarme RGL, c'était nickel. Cela ne fait plaisir qu'à moi... Par contre, si je fais le yoyo, et que sur l'axial offset le cour est déséquilibré, ce n'est pas un bon... Quand les courbes se suivent parfaitement entre la température moyenne 
et la température de référence [c'est bien]; si cela avait zigzagué, c'est dégueulasse... On suit 56 paramètres... C'est suffisant pour savoir si on pilote bien. Il y a des moments où cela sort du décor, parce qu'il y a des variations du réseau. Ce n'est pas "parfait»on tend vers l'idéal. (tableautiste nucléaire)

On voit à l'œuvre ici ce qu'Yves Clot et Daniel Faïta (2000: 14-18) ont désigné comme des «styles» dans le travail: «Chacun a sa petite touche personnelle. [C'est] comme sa voiture, chacun ne la conduit pas pareil. Certains vont être brutaux sur l'embrayage, d'autres auront une conduite plutôt cool» (tableautiste, nucléaire). Mais ce sont aussi les installations elles-mêmes qui sont pensées dans leur spécificité productive, certaines étant plus instables et donc plus difficiles à conduire que d'autres, plus névralgiques économiquement, ou plus dangereuses ou plus porteuses de risques environnementaux:

"C'est un des postes les plus durs. Qu'est-ce qu'il a de plus par rapport aux autres? C'est surtout la technicité du poste... Le poste est au milieu [du circuit productif]. S'il y a un souci à la salle, donc, il y a la technicité qui est assez importante. Il y a aussi les produits, c'est dangereux... Si on envoie du chlore gazeux dehors, cela ne va pas être sympa, ni pour nous, ni pour les gens qui sont dans l'usine, ni pour l'environnement! C'est un poste, si vous voulez, faut être attentif, comme dans n'importe quel secteur, mais où on a une période d'action très courte. (tableautiste, chimie)

Pour parler de leur activité, les tableautistes utilisent fréquemment un vocabulaire topographique, celui de la «ronde», qu'ils mènent sur leur écran en doublon de celle effectuée dehors par les opérateurs extérieurs. Les installations ne peuvent être conduites sans la construction de schémas mentaux plus ou moins idiosyncrasiques de leur fonctionnement (Hoc, 1996). Ces schémas sont nourris en permanence par les expériences vécues. Se trouver dans des situations délicates comme le démarrage ou l'arrêt d'installation, la baisse ou la montée de charge d'électricité (dans le nucléaire), ou la survenue d'incidents n'est pas seulement un aléa du métier, c'en est le cœur. C'est ce qui permet de gravir une échelle de prestige professionnel. Comme dans les sports à risque ou dans l'art militaire, ces situations sont donc à la fois redoutées et espérées, car c'est dans ces conditions risquées que les opérateurs ont vraiment l'impression de «conduire» les installations. Elles sont celles qui donnent du sens au travail. Ces moments d'intense activité, où l'opérateur sent l'installation réagir à ses commandes, relèvent de ce qu'Alexandra Bidet a appelé le «vrai boulot» (Bidet, 2011: 243-295), dans son étude des surveillants du réseau téléphonique:

Demain, on va baisser la charge. Ce sera super intéressant. Là je serai au primaire, je vais me faire plaisir. Lundi matin, on a remonté la charge, je me suis régalé. Dès que c'est technique, on aime bien. Mais être en réunion ou derrière le bureau, c'est la misère, cela ne m'intéresse pas, il n'en sort rien. Alors que là, on joue... Enfin, ce n'est pas le bon terme... on exploite une super-machine super-complexe où il y a beaucoup de paramètres techniques à vérifier. On a prise sur beaucoup de choses [...]. (tableautiste, nucléaire)

En prise directe avec les installations, un tout autre travail de surveillance est mené par les opérateurs extérieurs, aussi appelés rondiers ou agents de terrain. C’est le premier 
poste qu'occupent les opérateurs nouvellement embauchés avant de devenir éventuellement tableautistes. Le travail des rondiers est plus concret, plus en phase avec une réalité palpable, que celui des tableautistes. Ces deux registres de la connaissance des installations, du «dedans» et du «dehors», sont étroitement connectés grâce au dialogue constant que le tableautiste entretient avec son correspondant extérieur.

Le travail à l'extérieur se décline en trois modalités (Daniellou 1986; Palaci 2009): la première relève d'un travail routinier, prescrit dans les procédures, celui des «tournées » ou «rondes» régulières qui sont effectuées en moyenne à trois reprises par quart et dont la plus importante est réalisée en début de quart; la seconde consiste à effectuer des contrôles ou des opérations (fermeture/ouverture de vanne) à la demande des tableautistes lorsqu'une anomalie a été détectée ou d'accompagner sur le terrain les opérations de démarrage ou d'arrêt d'installation; la troisième consiste à préparer, en association avec les opérateurs en salle, les opérations de «consignation» des installations, c'est-à-dire leur mise hors circuit pour le travail de maintenance.

Lors des tournées, qui durent d'une demi-heure à deux heures, selon les secteurs et la situation, les rondiers réalisent plusieurs types d'opérations: prises d'échantillon, relevés de mesures sur les analyseurs chimiques, vérification de niveaux de pression, de remplissage des cuves et des bâches de stockage. Ils procèdent également à des contrôles de fuite afin de détecter des pannes ou des dérives de fonctionnement qui auraient échappé aux systèmes de contrôle accessibles depuis les écrans d'ordinateur. La manière de procéder aux rondes est variable, selon l'ancienneté de l'opérateur dans le secteur, l'humeur du jour, la situation météorologique (chaleur, froid, précipitations), la présence ou non de dispositifs fragiles à examiner de plus près, ou, encore, la marche générale de l'installation, qui peut connaître des périodes de fonctionnement calmes ou agitées. Les opérateurs organisent leur parcours et modulent la durée de leur ronde selon la combinaison de ces divers paramètres. L'enjeu du maintien d'un contrôle in situ est en effet l'imparfaite fiabilité des informations transmises par les automatismes dont disposent les opérateurs en salle.

L'opérateur extérieur traque les signes d'éventuels dysfonctionnements pour faire remonter l'information en salle de contrôle. Selon les sites, le chemin de ronde est plus ou moins long et acrobatique. Il faut parfois gravir les échelles protégées par des crinolines pour monter dans les tours. Les échantillons doivent être prélevés avec précaution, car les produits sont toxiques, corrosifs ou, simplement, à haute pression ou brûlants; l'ouverture des vannes, qui nécessite un engagement corporel, peut donner lieu, sous l'effet de la pression, à des projections de produit. Tous les sens sont mobilisés. La vue d'une auréole sur le sol ou de traces de tartre sur un robinet indique une fuite de liquide. L'ouïe permet d'identifier des bruits anormaux, sur les pompes par exemple (d'où les réticences fréquentes à se protéger les oreilles). L'odorat signale des fuites de gaz: si un gaz suffocant comme le chlore se détecte immédiatement, d'autres sont plus «vicieux», comme le chlorure de vinyle monomère à l'odeur de "pomme verte». L'odeur de bakélite, d'huile, de plastique chauffés fait soupçonner des risques d'incendie. Le contact physique avec les installations n'est pas non plus absent: toucher 
le palier d'une pompe fournit une estimation immédiate de sa température. En accompagnant les rondiers sur le terrain, nous avons pu aussi les observer poser leurs mains sur les cuves de stockage ou frapper dessus avec une clé pour évaluer leur niveau de remplissage.

La compétence du rondier repose aussi sur sa capacité à se repérer dans l'espace. L'expression d' "agent de terrain" prend ici tout son sens. Le bon agent de terrain est celui qui sait vite s'orienter pour trouver la vanne ou la pompe à manipuler. Dans les usines chimiques, les codes de couleurs des tuyaux aident au repérage dans l'espace: bleu pour l'eau, vert pour l'air ou l'azote, rouge pour les acides, rose pour l'hydrogène ou le dichloréthane, gris pour le gaz de chauffage, jaune pour le chlore ${ }^{11}$, mais cela ne garantit pas que l'on ne se perdra pas dans le réseau:

Quand j'ai commencé ma formation au chlore, un moment, je me suis aperçu que je suivais la rampe de l'escalier qui était jaune! Le tuyau que je suivais, il passait derrière une rambarde d'escalier, il descendait et... j'ai suivi l'escalier. Donc on se repère... mais des fois ça rate! (rire). On part de l'appareil et on lève la tête, on le suit. En gros tout le monde fait cela; en levant la tête, c'est très difficile d'arriver à savoir d'où ils viennent et où ils vont, il y a une foultitude d'embranchements et, effectivement, pour s'y repérer, c'est pas simple. (rondier chimie)

Parfois, les tuyaux, rouillés ou calorifugés, ne peuvent être distingués par leur couleur. Nombre d'autres repères sont toutefois présents sur le terrain. Ils ont souvent été ajoutés par les opérateurs, comme le font les marcheurs sur les chemins de randonnée: tuyaux et pompes marqués à la main de symboles (croix, flèches indiquant le sens du flux) ou de numéros. Dans la centrale nucléaire, nous avons également noté la présence de tels graffitis indigènes. Des consignes d'action (manière de tourner une vague par exemple) ou des alertes sur des installations défectueuses sont parfois également notées sur les tuyaux. Dans certains secteurs, des étiquettes officielles et normalisées ont été accrochées pour limiter le développement de ces inscriptions sauvages.

L'étude du travail des rondiers conduit à mettre en discussion la thèse, un peu abruptement admise par la plupart des sociologues qui ont travaillé sur l'automation, de la perte de contact avec la matière dans les industries à flux continus. Sans doute, la matière traitée est enfermée dans des tubulures et des réservoirs. Sa «mise à distance» est indispensable, puisque cette matière est en général plus ou moins toxique, corrosive ou brûlante. Mis à part le cas des prélèvements, quand la matière sort des tubulures, simples suintements, ou pire, expulsion brutale, c'est toujours l'indice d'un dysfonctionnement. Il n'est donc pas faux de dire que le rapport à la matière est médiatisé, même pour les opérateurs extérieurs. Mais c'est une erreur d'en conclure, comme tend à le faire Guy Jobert (2014), que le travail serait pour autant totalement «dématérialisé ». Le travail des opérateurs extérieurs engage leur corporéité et mobilise fortement leur sensorialité. À cet égard, on peut considérer qu'ils cherchent, précisément, à réta-

11. Ces peintures vives donnent, quand elles viennent d'être refaites, un air étonnamment gai à l'usine. Mais ce clinquant est parfois dénoncé par les opérateurs qui critiquent la fonction de «cache-misère » d'une peinture qui recouvre des tuyaux selon eux trop corrodés en leur cour. 
blir un contact direct avec la matière pour compenser les biais de traduction induits par les dispositifs de régulation qui alimentent le travail des opérateurs en salle.

La régulation d'ensemble de l'installation résulte de la combinaison complexe de ces deux prises, celle du dedans et celle du dehors. Les relations entre opérateurs extérieurs et intérieurs reposent sur un lien de dépendance réciproque qui exige un dialogue constant et délicat entre eux. Sur le terrain, le rondier est en permanence en contact par talkie-walkie ou téléphone avec son correspondant en salle, qui lui dit où mener ses investigations ${ }^{12}$. Certains dysfonctionnements, qui n’ont pas été repérés par les automatismes, ne sont pas signalés sur les écrans; réciproquement, certaines anomalies, qui apparaissent sur les écrans, restent incompréhensibles ou peuvent faire l'objet d'interprétations multiples, tant que l'on n'a pas croisé les informations électroniques et les relevés sensibles sur place. Selon le schéma cybernétique, le tableautiste représente le «cerveau» de la boucle de régulation. L'opérateur extérieur est quant à lui le «capteur» et, éventuellement, «l'effecteur», quand il ne se contente pas de signaler, mais agit matériellement:

L'agent de terrain, c'est les yeux, le nez, les oreilles de l'opérateur. Donc, on voit ce qu'il ne voit pas, c'est-à-dire tout ce qui n'est pas remonté en salle de commande. Lui, par rapport à ses écrans, il n'a pas la vue réelle. Une fuite, qu'elle soit de vapeur d'eau ou quoi que ce soit, si ce n'est pas une vraie grosse fuite, il ne la voit pas. (rondier nucléaire)

On dit souvent, un agent de terrain, c'est les yeux, les oreilles, et les mains de l'opérateur. C'est lui qui va détecter une petite fuite, parce que, moi, les fuites, je ne les vois pas. Il va dire: «Le palier de la pompe fait un bruit de callot; le roulement doit être mort...» On est complémentaire. (tableautiste nucléaire)

Si l'agent de terrain est soumis aux injonctions du tableautiste, le tableautiste est fonctionnellement dépendant de l'agent de terrain:

Notre travail? C'est guider le dedans car, dedans, on ne voit pas tout. (rondier, chimie)

Le produit qui sèche le dedans et qui rentre dans les manches, c'est pour enlever l'air du produit. Si cela se bouche et si cela commence à s'engorger, je ne le vois pas. Lui [l'opérateur extérieur], il le voit. Du coup, il arrive où il me demande de passer le Niro à l'arrêt à l'eau. Après, il va le déboucher. Moi, j'ai les yeux, mais, lui, il a les yeux ailleurs. Tout seul, je ne peux pas gérer le Niro. (tableautiste chimie)

Le degré d'autonomie des rondiers est variable suivant les secteurs. Plus le dispositif est automatisé, et plus le tableautiste est en mesure de le conduire seul depuis la salle de contrôle. Dans des installations moins automatisées, le rondier dispose de plus de marge de manœuvre.

La relation entre les deux membres de ce binôme n'est toutefois pas symétrique, car, c'est bien le tableautiste qui est «aux commandes». L'enjeu est donc dans la mise en place d'un subtil équilibre permettant d'installer discrètement l'autorité du tableau-

12. Dans les centrales nucléaires, où souvent les ondes ne "passent pas » en raison de l'épaisseur des murs, quand un téléphone n'est pas à proximité, et qu'il y a urgence, la communication se fait par la «sono », ce qui a pour effet, potentiellement stigmatisant, que tout un secteur de l'usine l'entend. 
tiste sans heurter la sensibilité du rondier. La soumission fonctionnelle des rondiers aux tableautistes est symboliquement renforcée par la hiérarchie salariale. Dans la carrière des opérateurs, les postes extérieurs précèdent les postes intérieurs. Aussi, les opérateurs extérieurs sont-ils en général classés à des coefficients plus faibles des conventions collectives. Même s'ils ne sont pas placés formellement sous les ordres des tableautistes, les rondiers sont donc bien soumis à leur hiérarchie fonctionnelle et symbolique $^{13}$. En fonction de sa connaissance de l'agencement spatial des installations et de sa capacité à ordonner les tâches à réaliser, l'opérateur intérieur facilitera plus ou moins le travail de son collègue extérieur. Le tableautiste doit, par exemple, avoir une bonne représentation des distances réelles entre deux points des installations car les vues des écrans qui représentent les installations ne sont pas isométriques. Du point de vue du rondier, le «bon tableautiste» est celui qui sait «économiser» son travail, c'està-dire, ne pas lui imposer inutilement des tâches fatigantes et dangereuses.

Longtemps, les rondiers ont été interdits de séjour dans les salles de contrôle. Dans son étude datant du début des années 1980, Gilbert de Terssac (1992) relatait que les rondiers, en principe encore exclus de la salle, venaient régulièrement s'y ressourcer pour confronter leurs observations à celles des tableautistes. Aujourd'hui, les rondiers sont régulièrement présents dans la salle de contrôle et dans la salle de repos attenante, qui constituent leur point d'attache entre leurs rondes. Chaque «retour» dans la salle, après une tournée régulière ou une mission motivée sur les installations, est l'occasion d'une confrontation des informations «du dedans» et du "dehors». Même si le tableautiste conserve le monopole de son clavier, il partage avec son rondier les informations qui émanent de son écran, de même que celui-ci lui livre celles qu'il a recueillies sur le site.

Tout se joue dans les règles informelles de l'échange et le choix du vocabulaire: «Il y en a c'est: "Va faire ci, va faire ça." Je préfere qu'on me dise: “Tiens, il y a cela qui ne va pas..." Que l'analyse se fasse en commun» (opérateur extérieur, chimie). L'important est que les demandes ne prennent pas (trop) la figure d'ordres. Dans cette configuration sociale d'évitement de la hiérarchie explicite, «il faut être superdiplomate», selon la formule d'un adjoint au chef de poste du nucléaire. La collaboration entre opérateurs intérieurs et extérieurs n'est pas toujours facile, en raison des décalages de représentation, mais aussi de la configuration matérielle des postes respectifs: le premier, face à son écran, dans son fauteuil ergonomique, est, au chaud en hiver et au frais en été, protégé des émanations toxiques ou des risques de radiation; le second grimpe aux crinolines par tous les temps (dans la chimie) ou s'expose potentiellement aux inhalations toxiques ou aux rayonnements ionisants (dans le nucléaire). Suivant leur tempérament, mais aussi le contexte, les rondiers peuvent donc recourir à des formes de «freinage» d'autant plus discrètes que les tableautistes ne savent pas exactement où ils sont: "Maintenant, c'est vrai qu'il y des postes qui sont tranquilles

13. Signalons toutefois l'organisation originale mise en place dans une des deux usines chimiques enquêtées, qui consistait à faire tourner les opérateurs, alternativement en salle et à l'extérieur, afin de supprimer toute hiérarchie entre intérieurs et extérieurs. 
et des postes où l'on travaille. Certains diront: “T'as pas vu, t'as pas entendu, donc t'as pas de manœuvres à faire." Ça, c'est des astuces. Si, moi, je dois faire une manœuvre..., bon, aller faire une manœuvre ou rester assis...»(rondier chimie préretraite). Toutefois, quand la sécurité est en jeu, la «diplomatie» peut battre en retraite.

\section{LE JOUR ET LA NUIT: QUI TIENT L'USINE ?}

Ajuster la production à une demande fluctuante dans des conditions satisfaisantes de sécurité et au coût optimal, telle est la mission affectée au travail quotidien dans une usine à flux continu. Mais les équipes doivent aussi être prêtes à gérer des crises d'importance plus ou moins grande. C'est pour satisfaire ce double objectif que des organisations complexes ont été mises en place. Pour assurer la surveillance en continu des installations, le travail est organisé en équipes postées qui se relèvent toutes les huit heures (quarts du matin, de l'après-midi et de la nuit) en suivant une «roulette». Tous les deux ou trois jours, l'équipe change de créneau horaire. Chaque équipe est composée d'une demi-douzaine d'opérateurs, intérieurs et extérieurs, encadrés par un chef de poste secondé par un ou deux adjoints.

Mais, sur l'organisation en quart, qui, pour reprendre une expression entendue dans le nucléaire, "gère le temps réel », se greffe une seconde organisation, inscrite dans une autre temporalité: celle du travail «à la journée» réalisé par les agents de maîtrise de jour (AMJ) et les ingénieurs de production ${ }^{14}$. Les agents de maîtrise de jour ont la responsabilité chacun d'une fraction des installations, dont le périmètre est en général plus restreint que celui d'une équipe postée ${ }^{15}$. Ils sont présents à des horaires «standards» du travail salarié (de huit heures du matin à cinq ou six heures de l'aprèsmidi) ${ }^{16}$. Ils fixent les consignes de marche en fonction de la production demandée, supervisent la planification et le suivi des travaux de maintenance; dans le nucléaire, certains ont aussi la responsabilité de planifier, en association avec les différents services techniques, les essais périodiques. Cette maîtrise de jour constitue un acteur-relais crucial entre «la production» (les équipes de quart) et «les services». Le personnel de jour comprend en effet en outre les administratifs et les services de maintenance chargés de la programmation et du contrôle du travail des entreprises sous-traitantes, lesquelles assurent l'essentiel du travail d'entretien, lorsque le site est en production ou lors des arrêts périodiques. Suivant les secteurs et les sites, les postés représentent entre

14. Dans le nucléaire, il y a des ingénieurs (chefs d'exploitation) chargés de la sécurité qui sont aussi de quart mais qui ne sont pas présents en salle de contrôle.

15. Autrement dit, un chef d'équipe posté aura en général comme référent deux agents de maitrise de jour. Les opérateurs, qui sont tous affectés à une zone géographique précise, n'auront affaire qu'à l'un d'entre eux. Un agent de maîtrise de jour suit, pour son secteur, l'activité de toutes les équipes postées qui se relaient sur une installation (cinq dans la chimie, sept dans le nucléaire).

16. Comme souvent les cadres, les ingénieurs chefs de service et les responsables de la sûreté (dans le nucléaire) sont parfois présents plus tard le soir. Dans les centrales nucléaires, un ingénieur est présent vingtquatre heures sur vingt-quatre. Une petite équipe d'ingénieurs est donc postée. En revanche, dans la chimie, les ingénieurs ne sont présents que le jour. Un ingénieur est toutefois toujours d' «astreinte», c'est-à-dire consigné à son domicile pour pouvoir se rendre au plus vite sur le site en cas d'incident important. 
vingt et trente pour cent du personnel propre des sites industriels, sans compter le personnel des entreprises sous-traitantes présent en permanence sur les sites, même en dehors des phases d'arrêt périodique ${ }^{17}$.

La distinction entre le «quart» et le «jour» est structurante dans toutes les industries de flux. Elle évoque celle qui oppose le «front» et l' «arrière» dans les armées en campagne, le navire et la «terre» dans la marine (Flécher, 2015) ou encore entre les opérateurs de vidéosurveillance et la hiérarchie administrative de la police municipale (Le Goff et al., 2011). Le personnel posté a le sentiment d'avoir l'usine en charge, d'être aux avant-postes en cas d'incident. L'expérience partagée de travail en horaires décalés, la vie en commun dans l'espace confiné des salles de contrôle crée, chez le personnel posté, un fort sentiment de solidarité collective. Celle-ci se manifeste et s'entretient à l'occasion des moments de convivialité auxquels sont très attachés les postés, comme la pause-café lors de la prise de poste où le traditionnel repas de nuit.

En exagérant la chose, les postés, c'est une famille. Normalement, le réfectoire, c'est un lieu d'isolement. De même que le vestiaire; on n'admet pas que des gens viennent jusque-là si on ne les invite pas. On n'aime pas, c'est quand on est en réunion [il fait allusion ici au rituel de la pause-café où sont échangées, lors de la relève entre équipes, des informations sur le quart passé et le quart à venir] pour parler des choses en privé. Si on voit débarquer quelqu'un, cela met mal à l'aise. (tableautiste chimie)

La métaphore de la famille ou de «l'équipe de foot» est d'ailleurs souvent évoquée pour souligner l'importance de la solidarité d'équipe:

Une équipe c'est comme une famille. Quand on dit: «Lui, il va changer d'équipe», c'est mal vécu [...]. Un A.M.P. [agent de maîtrise posté] qui passe A.M.J. [agent de maîtrise de jour], on dit qu'il «sort». «Il n'est plus de la famille.» (opérateur polyvalent chimie)

Dans mon équipe, j'essaye de restaurer une ambiance [...]. Il vaut mieux bosser avec quelqu'un que l'on apprécie. De toute façon, les incompétences des uns sont comblées par la compétence des autres. C'est comme dans une équipe de foot: tu peux avoir tous les meilleurs joueurs $d u$ monde, ce qu'on appelle la super feuille de match, et, s'ils ne s'entendent pas super bien... Alors que si tu as des joueurs moyens avec une super entente, s'ils vont se faire des passes, ils peuvent réussir à gagner. (opérateur de salle de contrôle, centrale nucléaire, 40 ans, niveau BTS d'électrotechnique)

La concurrence, la jalousie professionnelle, le jugement réciproque sur la compétence et la virtuosité existent, comme partout, mais leur expression doit rester discrète, tant les contraintes de la solidarité technique sont fortes au quotidien. Une des préoccupations principales des chefs de poste est de maintenir la convivialité au sein de l'équipe. Il faut s'assurer que les personnes n'hésitent pas à se parler, n'aient pas peur d'exprimer leur doute, de faire part d'éventuelles erreurs de conduite (Schön, 1994). À l'affût du moindre dérapage relationnel, ils contribuent activement à la construction de la cohé-

17. En phase de fonctionnement, le personnel sous-traitant présent sur les sites peut être évalué à environ un tiers du personnel propre. Lors des arrêts périodiques, ce personnel devient plus nombreux que le personnel propre. 
sion des équipes, à l'instar des officiers de marine étudiés par Serge Dufoulon (1998) ou des responsables du contrôle aérien étudiés par Gérard Dubey, dont l'autorité s'exerce, selon lui «dans des actions orientées vers l'équilibre du groupe, à l'image du chef indien décrit par Pierre Clastres, faiseur de paix et instance modératrice » (Dubey, 2006: 67-79).

La participation des chefs de poste aux moments de convivialité rituels, tant décriée par les techniciens et le personnel d'encadrement «de jour», est révélatrice à cet égard. L'enjeu en est la construction de la capacité collective de l'équipe. Le chef de poste doit être en mesure de prendre, chaque jour, «la température» de ses «troupes», pour décider à qui confier telle tâche délicate ou sur qui compter en cas de coup dur. On instaure la confiance en autorisant le dévoilement des inquiétudes et l'on facilite ainsi la circulation des informations nécessaires à la gestion du flux. Mais cette confiance n'est pas dissociable de la crédibilité technique dont jouissent les chefs de poste qui, régulièrement, «prennent la main» directement sur le processus, conseillent les opérateurs, et décident en dernier ressort de la conduite à tenir en cas de doute.

Comme dans une armée sur le champ de bataille, le rapprochement convivial des «chefs» et des «hommes» marque la condition commune relativement au danger partagé. En conséquence, les chefs de poste alimentent un système indulgent. En protégeant et en défendant «leurs» opérateurs contre les interventions de la hiérarchie de jour et en critiquant souvent sans ménagement, comme leurs hommes, l'action - ou l'inaction - de «ceux d'en haut», ils participent à la fabrication d'une solidarité des postés relativement aux «jours». Cette solidarité qui s'exprime par un discours fortement ouvriériste ( «eux» et «nous»), peut étonner l'observateur qui connaît le niveau moyen élevé de la qualification de ce personnel et, surtout, son salaire. Mais cette culture ouvriériste constitue le ciment social nécessaire dans des contextes industriels marqués par une forte dangerosité. Les contraintes de régulation interne des équipes poussent au repli centrifuge. Ce souci de cohésion, préoccupation première du chef de poste, est à la mesure de sa crainte de désagrégation du collectif de travail. Cette opposition est d'autant plus vive que, comme il en est de l'arrière pour l'armée ou, de la terre, pour la marine, le personnel de «jour» est, en moyenne, d'un niveau hiérarchique plus élevé et, fonctionnellement, en position de commanditaire à l'égard des postés.

De leur côté, les membres de l'encadrement technique de jour, soit les agents de maîtrise ou les ingénieurs, vivent mal la solidarité identitaire des postés qui se cristallise dans une opposition à eux. Car, eux aussi, ont le sentiment de "porter l'usine», en «assurant le fil rouge», selon une expression entendue à plusieurs reprises. Ils ne cachent pas leur irritation par rapport au monde du quart qui sous-estimerait selon eux leur utilité:

Les gens de quart, quand ils sont en quart, ont l'impression que ce sont eux qui font vivre la tranche. Non, ce n'est pas du tout cela... Ce sont les gens de journée qui programment tout au planning, qui se projettent dans l'avenir. Ils ont eu le passé, voient le présent et se projettent dans l'avenir. Les gens de quart n'ont pas de conscience que ce sont les gens de journée qui décident ce que la tranche fera. (opérateur en formation pour devenir AMJ, hors quart nucléaire) 
Dans une représentation aux antipodes de celle des postés, ils considèrent que ce sont eux qui, en fait, «tiennent» véritablement l'usine dans le temps:

Le passage hors quart permet de voir toute la tripaille qui a derrière. C'est un monde à part. Quand tu es en quart, tu viens à six heures, tu pars à treize heures... ta journée est terminée... Il y a des semaines, tu as onze jours de repos, tu as du temps libre. Et puis, hop, tu sais que tu as fini ton quart, tu as fait ton compte-rendu, le boulot fini ou pas fini... Quand tu es hors quart, tu t'en vas, ton boulot n'est pas fini; tu reviens, il est toujours pas fini. (agent de maîtrise de jour, nucléaire)

La position des agents de maîtrise de jour est d'autant plus fragile que leur situation hiérarchique supérieure ne se traduit pas dans l'échelle salariale effective, en raison de l'importance des «primes de poste» dont bénéficient leurs collègues de quart. De fait, beaucoup de salariés à la journée sont d'anciens postés, qui, avec l'âge, en raison de l'accumulation de fatigue et des contraintes de la vie familiale, sont «passés à la journée». L'ouvriérisme des postés, qu'ils considèrent volontiers comme des "privilégiés», en comparaison, par exemple, des «vrais» ouvriers, ceux des équipes des entreprises sous-traitantes, les insupporte donc:

$C A P, B E P, B A C, B T S$, ils sortent de l'école, ils viennent ici en poste et, un poste où, malgré tout, quand l'installation marche bien, ils se reposent. Les gens qui travaillent sur un chantier, ils ne savent pas ce que c'est... Moi, j'ai fait des chantiers. Alors, quand je leur dis qu'ils font attendre les mecs parce qu'ils boivent un café ou fument une cigarette une demi-heure ou une heure, alors que le mécano doit rendre des comptes par rapport au travail qu'il a fait... Comment il fait, le mécano? Ils ne se rendent pas compte de cela. Moi je dis, disons, ils ne sont pas conscients. Je ne dis pas c'est des nantis mais... (agent de maîtrise de jour, chimie)

Dans ce contexte, les phases d' "heures ouvrées» (telle est l'expression consacrée), c'està-dire entre huit heures du matin et cinq heures l'après-midi, où cohabitent dans l'usine l'équipe de quart et l'équipe de jour, sont tout à la fois marquées par la tension entre les deux catégories de personnel et par un échange intense entre elles, lequel est indispensable au fonctionnement des installations. Chacun va pouvoir confronter son point de vue à celui des autres, demander des précisions, expliciter les problèmes en face-à-face, lever éventuellement des malentendus, et des compromis ou des arbitrages pourront être dégagés. Ce quart de jour est le plus pénible pour l'équipe postée, qui perd la pleine maîtrise des installations dont elle dispose pendant les deux autres quarts et qui se sent plus ou moins «fliquée». Durant cette phase, les agents de maîtrise de jour, qui, en principe, ne devraient pas intervenir directement auprès des opérateurs, peuvent d'ailleurs se laisser aller à le faire, comme pour reprendre prise sur un processus qui leur a échappé depuis qu'ils sont passés «à la journée».

\section{CONCLUSION : ÉVANESCENCE DU TRAVAIL ET CONTRÔLE DES HOMMES}

Dans l'industrie de flux, les tâches ne sont pas étroitement définies, comme elles peuvent l'être, par exemple, dans les industries de montage métallurgique, dont l'emblématique secteur de construction automobile nous a servi de point d'appui comparatif au début de cet article. Il y règne ce que Pierre Naville a pu appeler une 
«distribution mobile de fonctions intégrées» (Naville, 1963: 117) Pour autant, la division du travail y est nettement marquée dans un jeu de distribution des responsabilités qui est tout à la fois technique et social. Les marqueurs identitaires y sont forts: l' «intérieur» et l' "extérieur» le «jour» et la «nuit». Les travaux sont étroitement interdépendants et, pourtant, passablement invisibles aux yeux des autres. Comment le tableautiste peut-il mesurer la vigilance du rondier lors de sa tournée? Comment inversement le rondier peut-il savoir si c'est à bon escient que le tableautiste le fait courir à l'autre bout de l'installation? Comment l'agent de maîtrise de jour peut-il contrôler la qualité du travail des équipes de quart sous sa responsabilité? Comment inversement l'équipe postée peut-elle évaluer la pertinence des opérations que la maîtrise de jour lui enjoint de mettre en œuvre?

Dans ce jeu de cache-cache, chacun tend à reprocher à l'autre de n'être pas là où il l'attend, ou, à l'inverse, d'être trop présent là où on aimerait rester entre soi. La faible visibilité du travail de l'autre — de l'opérateur à l'ingénieur — sur ce qui a été réellement fait résulte du découpage spatio-temporel des activités mais aussi de leur nature même, de leur caractère évanescent. Ainsi, un tableautiste qui regarde ses écrans d'un air détaché peut être considéré comme en état d'oisiveté par un ingénieur ou un agent de maîtrise de jour. D’où la critique récurrente de la faible vigilance des postés qui émane de l'encadrement de jour et la critique symétrique, par les postés, de l'improductivité du personnel de jour, qui prendrait plaisir à entraver leur travail en leur imposant des activités stériles.

Ces tensions constitutives de l'organisation professionnelle dans ces industries génèrent des confrontations de point de vue et des redondances, qui provoquent l'irritation au quotidien et soudent de façon réactive les identités professionnelles, mais qui, dans le même temps, contribuent de façon essentielle à la sécurisation de la gestion du flux. Ainsi, alors que le travail humain, tel qu'on se le représente communément, devient évanescent, il est au cœur de la préoccupation de toute l'organisation. En effet, personne ne peut concevoir son travail indépendamment du travail des autres. Surveiller les flux, c'est donc aussi contrôler les hommes, et ceci vaut entre pairs, comme dans toute la chaîne hiérarchique.

\section{RÉSUMÉ}

Comprendre en quoi et comment une activité, dite de travail, est productive et socialement reconnue comme telle, est indispensable pour penser les modes d'encadrement social de cette activité, au niveau de l'atelier, de l'entreprise, de la société tout entière. Encore faut-il pour cela saisir le travail dans sa technicité. Telle est la démarche que nous allons développer ici, en partant d'une analyse du travail de surveillance-contrôle observé dans différents sites chimiques et nucléaires pour penser, les problèmes de l'organisation salariale dans ce type d'entreprises.

Mots clés: automation, chimie, nucléaire, risque industriel, surveillance-contrôle

\section{ABSTRACT}

Understanding in what way and how a so-called work activity is productive and socially recognized as such is essential to describe the mode of social regulation of this activity, within the workshop, 
the company, and the entire society. But to achieve this, the technicality of work needs to be grasped. This is the approach that we shall develop here, starting with an analysis of monitoring and control work observed in different chemical and nuclear facilities, in order to reflect on the problems of salary organization in this kind of company.

Key words: automation, chemistry, nuclear, industrial hazard, monitoring and control function

\section{RESUMEN}

Comprender en qué forma y cómo una actividad, enunciada como trabajo, es productiva y socialmente reconocida como tal, resulta indispensable para pensar los modos de enmarcamiento social de dicha actividad a nivel del taller, de la empresa, de la sociedad en su conjunto. Para ello, es necesario comprender el trabajo a nivel de su tecnicidad. En este artículo desarrollamos este proceso, partiendo de un análisis del trabajo de supervisión y control observado en diferentes emplazamientos químicos y nucleares, con el fin de pensar los problemas de la organización salarial en este tipo de empresas.

Palabras clave: automatización, química, nuclear, riesgo industrial, supervisión-control

\section{BIBLIOGRAPHIE}

Amalberti, R. (1996), La conduite de systèmes à risques, Paris, Presses universitaires de France.

Avenel, G. (1895), «Le mécanisme de la vie moderne (VII)», Revue des deux mondes, tome 132, p. 521-552. Bidet, A. (2007), «La mise en récit du trafic téléphonique. Prendre prise sur un travail «immatériel», Raison Présente, $\mathrm{n}^{\circ} 162$, Nouvelles formes de travail et d'emploi.

Bidet, A., Borzeix, A., Pillon, Th., Rot, G. et Vatin, F. (2006), Sociologie du travail et activité, Toulouse, Octarès.

Bidet, A. (2011), L'engagement au travail, Paris, PUF.

Bidet, A. et Vatin, F. (2016), «Travailler, c'est produire. Activité, valeur, et ordre social», in Dujarier, M.-A., Gaudart, C., Gillet, A. et Lenel, P. (dir.), L'activité en théories. Regards croisés sur le travail, Toulouse, Octarès.

Blauner, R. (1964), Alienation and Freedom. The factory worker and his industry, Chicago, University of Chicago Press.

Bourrier, M. (1999), Le nucléaire à l'épreuve de l'organisation, Paris, PUF.

BRIGHT, J. R. (1958), Automation and management, Boston, Harvard University.

Cellier, J.-M. (1996), «Exigences et gestion temporelle dans les environnements dynamiques», dans J.-M. Cellier, V. de Keyser et C. Valot (dir.), La gestion du temps dans les environnements dynamiques, Paris, PUF, p. 19-48.

Chateauraynaud, F. (1997), «Vigilance et transformation, Présence corporelle et responsabilité dans la conduite des dispositifs techniques», Réseaux, n 85, p. 101-127.

Сьот, Y. et al. (2000), «Genre et styles en analyse du travail. Concepts et méthodes», Travailler, n 4, p. 7-36.

Daniellou, F. (1986), L'opérateur, la vanne, l'écran. L'ergonomie des salles de contrôle, Montrouge, Éditions de l'ANACT, coll. «Outils et méthodes».

Diebold, J. (1952), Automation: The advent of the automatic factory, New York, Van Nostrand.

Dodier, N. (1993), Les hommes et les machines. La conscience collective dans les sociétés technicisées, Paris, Métailié.

Doniol-Shaw, G. et al. (1995), Les intermittents du nucléaire, Toulouse, Octarès.

DRUCKer, Peter (1957), Pratique de la direction des entreprises, Les éditions d'organisation.

Dubey, G. (2006), «Autonomie et hétérogénéité sociale dans les grands systèmes techniques», in Bidet, A. et al. (dir.), Sociologie du travail et activité, Toulouse, Octarès, p. 67-79. 
Duclos, D. (1987), «La construction sociale du risque: le cas des ouvriers de la chimie face aux dangers industriels », Revue française de sociologie, XXVII, p. 17-42.

Dufoulon, S. (1998), Les gars de la marine. Ethnographie d'un navire de guerre, Paris, Métailié.

FLÉCHER, C. (2015), Navigations humaines au gré du flux mondialisé. Le travail des marins de commerce sur les navires français de nos jours, Thèse pour le doctorat de sociologie, Université de Paris Ouest Nanterre.

Foote Whyte, W. (1994), Participant Observer. An autobiography, Chap. 16 «Oklahoma and the Philipps Petroleum Cie», Cornell University NY, p. 126-127.

Foote Whyte, W. (1955), «Ingineers and Workers: A Case Study», Human Organisation, vol. 14, n 4, p. 3-12. Fournier, P. (2012), Travailler dans le nucléaire, Paris, Armand Colin.

Galle, R. et Vatin, F. (1981), «Production fluide et ouvrier mobile. Procès de production et organisation du travail dans le raffinage pétrolier», Sociologie du travail, vol. 23, n 3, p. 275-292.

Gallie, D. (1977), «Automatisation et légitimité de l’entreprise capitaliste», Sociologie du travail, n³, p. 221-242.

Gallie, D. (1978), In Search of the New Working Class, Cambridge, Cambridge University Press.

Hoc, J.-M. (1996), Supervision et contrôle de processus. La cognition en situation dynamique, Grenoble, PUG. Jobert, G. (2014), Exister au travail. Les hommes du nucléaire, Paris, Érès.

Maurice, M. (1980), «Le déterminisme technologique dans la sociologie du travail (1955-1980). Un changement de paradigme?", Sociologie du travail, $\mathrm{n}^{\circ} 1$, p. 22-37.

Maurice, M., Sellier, F. et Silvestre, J.-J. (1982), Politique d'éducation et organisation industrielle en France et en Allemagne: essai d'analyse sociétale, Paris, PUF.

Meda, D. (1995), Le travail, une valeur en voie de disparition, Paris, Aubier.

Mumford, L. (1950), Technique et civilisation, Paris, INSEE, coll. «Esprit. La Cité prochaine».

Le Goff, T., Malochet, V. et Jagu, T., Surveiller à distance. Une ethnographie des opérateurs municipaux de vidéosurveillance, IAU-IDF, p. 62, 20.

Naville, P. (1963), Vers l'automatisme social?, Paris, Gallimard.

Palaci, F. (2009), Les agents de terrain du nucléaire: comprendre leur activité pour contribuer à la conception d'un outil d'aide informatique, Mémoire de master professionnel d'ergonomie, Paris, Conservatoire national des arts et métiers.

Rот, G. et VAtin, F. (2016), «Ici, on ne fabrique pas des marshmallows» : peur de l'accident et peur de mal faire dans les industries de flux à haut risque», dans Guenette, Alain-Max et al. (dir.), La peur au travail, Toulouse, Octarès, coll. «Le travail en débats», série Colloques \& Congrès, p. 155-175.

Rot, G. et VAtin, F. (2015), "Ingénieurs et sociologues face à l'automation: le cas Renault 1945-1960", dans Serge Benoît et Alain Michel (dir.), Le monde du génie industriel au Xx siècle: autour de Pierre Bézier et des machines-outils, Belfort, Presses de l'UTBM, p. 195-206.

Rot, G. et Vatin, F. (à paraître), Au fil du flux.

Rот, G. (2007), «Le travail de contrôleur de flux, de Pierre Naville à Alain Resnais. Propos sur le Chant du styrène (1958)", Revue européenne d'histoire sociale, Revue européenne d'histoire sociale — Histoire \& Sociétés, décembre 2007, Dossier Pierre Naville: mesure et logique du social, $\mathrm{n}^{\circ} 24$.

Rот, G. (2002), «Fluidité industrielle, Fragilité organisationnelle», Revue française de sociologie, $\mathrm{n}^{\circ} 43, \mathrm{n}^{\circ} 4$, p. 711-737.

Rот, G. (2006), Sociologie de l'atelier. Renault, le travail ouvrier, le sociologue, Toulouse, Octarès.

SHön, D. (1983), The reflexive practionner. How professionals Think in Action, Basic Books.

Terssac, G. (1992), Autonomie dans le travail, Paris, PUF.

Touraine, A. (1955), L'évolution du travail aux usines Renault, Paris, CNRS.

VAtin, F. (1987), La fluidité industrielle, Paris, Méridiens Klincksieck.

VAtin, F. (2007), «Le travail: contrainte productive, contrainte salariale», in François Vatin (dir.), Le salariat. Théorie, histoire et formes, Paris, La Dispute.

Vatin, F. (2014), Le travail. Activité productive et ordre social, Paris, Presses universitaires de Paris Ouest.

Woodward, J. (1965), Industrial organization: theory and practice, Oxford University Press.

Walker, Ch. R. (1957), Toward the Automatic Factory: A Case Study of Men and Machines, New Haven, Yale University Press. 